\title{
Statistical Approach to the Cosmological-Constant Problem on Brane Worlds
}

\author{
F. K. Diakonos and E. N. Saridakis * \\ Department of Physics, University of Athens, GR-15771 Athens, Greece
}

\begin{abstract}
We investigate the physically accepted solutions of general Braneworld scenarios, scanning uniformly the associated parameter space. Without making any further assumptions we find that solutions which give "small" Hubble parameters on the physical brane, and therefore "small" effective cosmological constants on the $4 D$ Universe, are far more probable than those with "large" ones. Eventually, their distribution tends to the $\delta$-function in the limit of continuous covering of the parameter space.

PACS numbers: 98.80.-k, 04.50.-h, 02.60.Cb
\end{abstract}

Why is the cosmological constant of the observable Universe many orders of magnitude smaller than expected within the context of ordinary gravity and quantum field theory? Since Einstein (1917) many authors have tried to give a plausible and coherent answer ([1] and references therein). Most of the earlier attempts were within the general relativity context, while other authors were led to the obscure anthropic principle [2]. An outlet of different origin arose since Zumino showed that unbroken supersymmetry leads to vanishing vacuum energy [3]. Unfortunately, and despite many attempts in the context of supergravity (or non-conventional gravity) [4, 5] and superstrings 6], in the real world we know of no mechanism that can produce an effective cosmological constant within the remarkable small experimental limits, and especially to keep it unaffected by quantum fluctuations. Another interesting approach was followed by Hawking and later by Coleman [7], showing that a distribution of values for the effective cosmological constant with an enormous peak at zero could arise. Although the whole approach raises many doubts 1], the idea of restricting the cosmological constant to small values through statistical considerations is interesting and consists the basis of the present work. Finally, the approaches to the problem which remain in the conventional $4 \mathrm{D}$ framework suffer from loss of generality [8] , and the use of cosmological observations on behalf of small values pays the price of adding various non-general assumptions [9].

The discussion was reheated after the arrival of observational data indicating that there is a positive cosmological constant in the universe 10]. At the same time the Braneworld scenario appeared in the literature [11]. Amongst other consequences, it offered new paths of producing a zero or small (effective) cosmological constant on the physical brane consisting our Universe [12, 13], but none could offer a robust and undoubted justification. Most of these works re-introduced in the new Braneworld context (of various versions and dimensions) the old finetuning procedure of conventional cosmology.

\footnotetext{
*E-mail: msaridak@phys.uoa.gr
}

In conclusion, there is not a convincing and general solution to the cosmological constant problem. Definitely, a complete apprehension will arise from a fundamental theory of nature and the breaking of its symmetries. Since this theory is unknown at present, effective or even phenomenological descriptions are probably preferable, although incomplete. The minimum requirement is that of remaining general and fine-tuning free. In this Letter we follow this direction in the context of Braneworld scenario, and we explore the physically accepted solutions in the parameter space, without making any additional assumptions. Obviously this approach cannot act as a solution to the cosmological constant problem. However, it can be enlightening and provide some arguments for its possible elaboration in statistical terms.

We consider a general class of Braneworld models, characterized by the action:

$$
\begin{gathered}
\kappa_{5}^{2} S=\frac{1}{2} \int d^{4} x d y \sqrt{-g} R+\int d^{4} x d y \sqrt{-g}\left[-\frac{1}{2}(\partial \phi)^{2}-V(\phi)\right] \\
-\sum_{i=1,2} \int_{b_{i}} d^{4} x \sqrt{-\gamma}\left\{[K]+U_{i}(\phi)\right\},
\end{gathered}
$$

where $\kappa_{5}^{2}=\frac{1}{M_{5}^{3}}$ is a $5 D$ gravitational constant, and all quantities are measured in units of $M_{5}$. The first term describes gravity in the five dimensional bulk space, the second term corresponds to a minimally coupled bulk scalar field with the potential $V(\phi)$ and the last term corresponds to two $3+1$ dimensional branes, which constitutes the boundary of the $5 D$ space. We include a potential term $U(\phi)$ for the scalar field at each of the two branes, denoting with $\gamma$ the associated induced metric and with $K$ its extrinsic curvature. The square brackets denote the jump of any quantity across a brane $([Q] \equiv$ $\left.Q\left(y_{+}\right)-Q\left(y_{-}\right)\right)$. Assuming $S^{1} / \mathbb{Z}_{2}$ symmetry across each brane we restrict our interest only in the interbrane space $[13,14,15]$, (we use $\left[Q^{\prime}\right]_{0}=2 Q^{\prime}\left(0^{+}\right),\left[Q^{\prime}\right]_{1}=-2 Q^{\prime}\left(1^{-}\right)$). As usual the two branes are taken parallel, $y$ denotes the coordinate transverse to them and isometry along three dimensional $\mathbf{x}$ slices including the branes, is assumed. For the metric we choose the conformal gauge [15]:

$$
d s^{2}=e^{2 B(t, y)}\left(-d t^{2}+d y^{2}\right)+e^{2 A(t, y)} d \mathbf{x}^{2} .
$$

This metric choice, along with the residual gauge free$\operatorname{dom}(t, y) \rightarrow\left(t^{\prime}, y^{\prime}\right)$ which preserves its form, allows 
us to "fix" the positions of the branes. Without loss of generality we can locate them at $y=0,1$, having in mind that their physical distance at a specific time is given by $D(t)=\int_{0}^{1} d y e^{B(t, y)}[15$. The non-trivial fivedimensional Einstein equations consist of three dynamical:

$$
\begin{array}{r}
\ddot{A}-A^{\prime \prime}+3 \dot{A}^{2}-3 A^{2}=\frac{2}{3} e^{2 B} V \\
\ddot{B}-B^{\prime \prime}-3 \dot{A}^{2}+3 A^{\prime 2}=-\frac{\dot{\phi}^{2}}{2}+\frac{\phi^{2}}{2}-\frac{1}{3} e^{2 B} V \\
\ddot{\phi}-\phi^{\prime \prime}+3 \dot{A} \dot{\phi}-3 A^{\prime} \phi^{\prime}+e^{2 B} V_{, \phi}=0,
\end{array}
$$

and two constraint equations:

$$
\begin{array}{r}
-A^{\prime} \dot{A}+B^{\prime} \dot{A}+A^{\prime} \dot{B}-\dot{A}^{\prime}=\frac{1}{3} \dot{\phi} \phi^{\prime} \\
2 A^{\prime 2}-A^{\prime} B^{\prime}+A^{\prime \prime}-\dot{A}^{2}-\dot{A} \dot{B}=-\frac{\dot{\phi}^{2}}{6}-\frac{\phi^{2}}{6}-\frac{1}{3} e^{2 B} V,(4)
\end{array}
$$

where primes and dots denote derivatives with respect to $y$ and $t$ respectively. Additionally, from the boundary terms in the action for the branes we obtain the following junction (Israel) conditions:

$$
\left[A^{\prime}\right]=\mp \frac{1}{3} U e^{B}, \quad\left[B^{\prime}\right]=\mp \frac{1}{3} U e^{B}, \quad\left[\phi^{\prime}\right]= \pm e^{B} U_{, \phi},
$$

where the upper and lower signs refer to the branes at $y=0,1$, respectively. For the bulk and brane potentials we assume:

$$
V(\phi)=\frac{1}{2} m^{2} \phi^{2}+\Lambda, \quad U_{i}(\phi)=\frac{1}{2} M_{i}\left(\phi_{i}-\sigma_{i}\right)^{2}+\lambda_{i} .
$$

This is a general form for $V(\phi)$, consistent with the brane stabilization mechanism [16], $\Lambda$ being the $5 D$ bulk cosmological constant. For $U_{i}(\phi)$ the quadratic form is also general [15], incorporating the brane tensions $\lambda_{i}$ as well as the value $\phi_{i}$ of $\phi$ on the i-th brane. Finally, the induced $4 D$ metrics of the two ("fixed"-position) branes in the conformal gauge are simply given by: $d s^{2}=$ $-d \tau^{2}+a^{2}(\tau) d \mathbf{x}^{2}$, with $\tau_{i}=e^{B_{i}} t$ and $a_{i}=e^{A_{i}}$ the proper times and scale factors of the two branes [17]. Thus, for the Hubble parameter on the branes we acquire

$$
\left.H_{i} \equiv \frac{1}{a} \frac{d a}{d \tau}\right|_{i}=e^{-B_{i}} \dot{A}_{i},
$$

and we identify the physical brane with the one at $y=0$.

The described model is quite general and includes the full spacetime evolution of the $5 D$ Braneworld. In order to investigate some of its consequences we first examine the stationary sub-class of solutions, assuming that:

$$
B(t, y) \rightarrow B(y), \quad A(t, y) \rightarrow B(y)+H t .
$$

This choice leads to a fixed bulk geometry ( $\phi$ is time independent), and maximally symmetric (de Sitter or Minkowski) branes: $d s^{2}=e^{2 B(y)}\left(d y^{2}-d t^{2}+e^{2 H t} d \mathbf{x}^{2}\right)$, where the Hubble parameter on the physical brane is simply $H_{0}=e^{-B(0)} H$. If we set $V(\phi)=0$ we can acquire analytical solutions for the system of equations (3) and (44), depending on the sign of $H^{2}$. For $H^{2}>0$ and setting $H=\left|\sqrt{H^{2}}\right|$ we get:

$$
\begin{array}{r}
B(y)=B(0)+\frac{1}{3} \log \left(\frac{B^{\prime}(0)}{H} \sinh 3 H y+\cosh 3 H y\right) \\
\phi(y)=\phi(0)-\frac{2}{\sqrt{3}} \log \left[\frac{\left(e^{3 H y}-u\right)(1+u)}{\left(e^{3 H y}+u\right)(1-u)}\right]
\end{array}
$$

where $u=\sqrt{\frac{B^{\prime}(0)-H}{B^{\prime}(0)+H}}$, and always $\left|B^{\prime}(0)\right| \geq H$ for the existence of a solution. When $H^{2}<0$ and setting $\theta=\left|\sqrt{-H^{2}}\right|$, the solutions can be obtained from (9) under the replacement $H \rightarrow i \theta$ [18]. We mention that we do not make additional assumptions, excluding this case $\left(H^{2}<0\right)$ by hand, since we investigate any possible solution. Finally, expressions (9) have to satisfy the boundary conditions (5), and eventually $B(0), B^{\prime}(0)$ and $\phi(0)$ and $H$ are given in terms of the six parameters of the model $\left(M_{0}, \lambda_{0}, \sigma_{0}, M_{1}, \lambda_{1}, \sigma_{1}\right)$.

In order for the solutions to be physically accepted, we have to assure that no "naked" singularities are present in the physically meaningful interbrane space, i.e between $y=0$ and $y=1$. As it is shown in [19], the singularities of the aforementioned solutions are indeed true (and not just coordinate ones) since they possess incomplete geodesics reaching a point of divergent curvature. An equivalent way to reveal the true nature of these singularities, is by calculating explicitly any of the 5D curvature invariants such are the Ricci scalar, the Kretschmann scalar and the Weyl tensor, and showing that they diverge. This has been performed in [5, 15, 20], and has been straightforwardly verified by us that these curvature invariants do diverge in the singularities of the solutions (9), offering a robust proof of their true (naked) nature.

For $H^{2}<0$ case, the requirement of singularity absence forces the physically accepted solutions to satisfy:

$$
3 \theta-\pi<\arctan \left(-\frac{\theta}{B^{\prime}(0)}\right)<0 .
$$

Thus, this relation provides a narrow and absolute window for $\theta$, excluding areas of the $6 D$ parameter space leading to $\theta$-values larger than $\pi / 3$. For the $H^{2}>0$ case the avoidance of singularities leads to:

$$
H<\left|B^{\prime}(0)\right|<H \operatorname{coth}(3 H),
$$

and since $B^{\prime}(0)$ and $H$ are functions of the six parameters of the model, the window (11) gives the limits of the sub-space of the $6 D$ parameter space that corresponds to accepted solutions. Inequality (11) becomes actually an equality for large $H$ values $(H \gtrsim 2$ in the units we use) since both edges practically coincide. The resulting relation acts as a constraint in the $6 D$ parameter space, 
and therefore the parameter sub-space which corresponds to physically accepted solutions with large $H$ is of lower dimensionality, i.e it has been reduced to a sub-surface:

$$
H=\left|B^{\prime}(0)\right|=H \operatorname{coth}(3 H) .
$$

In conclusion, from the above analytical calculation it is implied that the demand for absence of divergences in the solutions restricts the probable $H^{2}$ values (and therefore $H_{0}^{2}$ ones) to a narrow window around zero (solutions with large $H$ have zero measure). The additional requirement of fulfilling the junction conditions on the second brane makes the parameter sub-space, leading to a solution, in-homogenous and non-compact [14].

Let us verify numerically the acquired results of the stationary case with zero bulk potential. The main difficulty of solving the equation system (3)-(6) is that one has to satisfy the constraints (4) and boundary conditions (51) at $t=0$, ensuring that no divergencies are present in the interbrane space, task which proves to be extremely hard in general. The restriction to $t=0$ is easily justified since the dynamical equations (3) preserve the constraints (4), and furthermore for the stationary case at hand the boundary conditions (5) are time-independent. The method we use is the following: We first choose randomly the values of the 6 model parameters, uniformly distributed in a $6 \mathrm{D}$ hyper-cube. The obtained results do not depend on the hyper-cube's size, but on its effectual covering (number of parameter multiplets used in the calculation). We use the globally convergent SchmelcherDiakonos algorithm 21] to solve the transcendental equation system (4)-(5), with accuracy $10^{-13}$. Thus, all existing solutions are easily obtained, which is not the case if one uses Newton's method [15], where a good initial guess, of a (relatively small) interval containing the root to be found, is needed as an input. We find hexads of parameters corresponding to acceptable solutions, and calculate $H_{0}$ through $H_{0}=e^{-B(0)} H$. In fig. 19 we present the $H_{0}^{2}$ histogram for $N=10^{6}$ accepted solutions, and we observe that $\rho\left(H_{0}^{2}\right)$ is strongly restricted around zero according to windows (10) and (11). A numerical fit specifies that for $H_{0}^{2}>0$ the aforementioned histogram is an exponential (coefficient of determination $R^{2} \approx 0.999$ ):

$$
\rho\left(H_{0}^{2}\right) \sim e^{-\alpha H_{0}^{2}},
$$

with exponent $\alpha \approx 3$. In order to provide an additional verification, in fig. 1 b we depict $H$ versus $B^{\prime}(0)$ for solutions that have $H^{2}>0$. We observe that for small $H$ $(H<0.5)$ inequality (11) holds, and it gradually transforms to equality (12) as $H$ grows, i.e the parameter subspace that corresponds to accepted solutions becomes thiner and thiner and finally results in a sub-surface.

We mention here that in our analysis we follow the "anti-fine-tuning" approach to the problem, determining first, unbiased (i.e randomly), the six model parameters and then investigating the solution features. This is opposite to the usual method in the literature, which is to

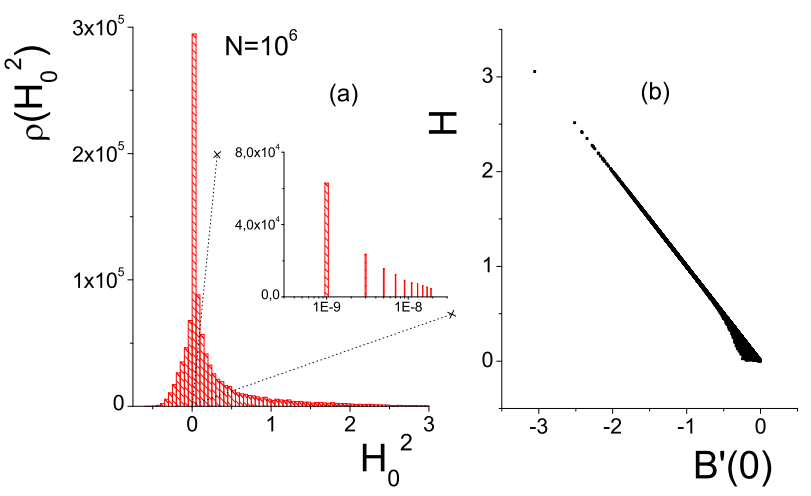

FIG. 1: (Color online) In graph a) we present the $H_{0}^{2}$ histogram for the accepted solutions. In graph b) we depict $H$ vs $B^{\prime}(0)$ for solutions with $H^{2}>0$. Both graphs correspond to the stationary case with zero bulk potential, and each edge of the parameter space hyper-cube extends from $-10^{3}$ to $10^{3}$.

fulfil the boundary conditions in the first brane at $y=0$, choose an arbitrary $H$, solve the constraints (4) towards $y=1$ and then fit the brane parameters of the second brane so that the corresponding junction conditions are fulfilled. Definitely this fine-tuning procedure has the disadvantage of neglecting several solutions, allowing for an apparent uniform distribution of the $H$ value, contrary to the fact that the considered solution space is a sub-surface of the full $6 D$ parameter space. The source of the misleading behavior is the over-determined nature of the problem, i.e the existence of more relations and requirements to be fulfilled (including conditions that are not expressible through a strict equation, such is the absence of naked singularities) than the number of model parameters. In such cases our method is better in order to reveal the full characteristics of the parameter space and its sub-space that corresponds to solutions.

After this verification of the analytical calculations we can proceed to numerical solutions for the stationary case with full bulk potential. Again, we randomly choose the values of the 8 model parameters $\left(M_{0}, \lambda_{0}, \sigma_{0}, M_{1}, \lambda_{1}, \sigma_{1}\right.$, $m$ and $\Lambda$ ), from a uniform distribution, and we solve the transcendental equation system (4)-(5). We mention here that we do not use any a priori restrictions on the parameter values arising from physical arguments (for example $\Lambda<0$ in order to acquire a $5 D$ AdS bulk as required by many authors), since we want to be as general as possible. In fig. 2a we present $\rho\left(H_{0}^{2}\right)$ for $N=10^{6}$ such solutions, and a numerical fit for $H_{0}^{2}>0$ gives again an exponential $\left(R^{2} \approx 0.998\right)$ of the form (13) with exponent $\alpha \approx 2.9$. Although an analytical treatment is not possible in this case, the qualitative results are similar to those obtained for zero bulk potential. Indeed, it seems that "small" $H_{0}$ arise from a (non-homogenous and non-compact) sub- 


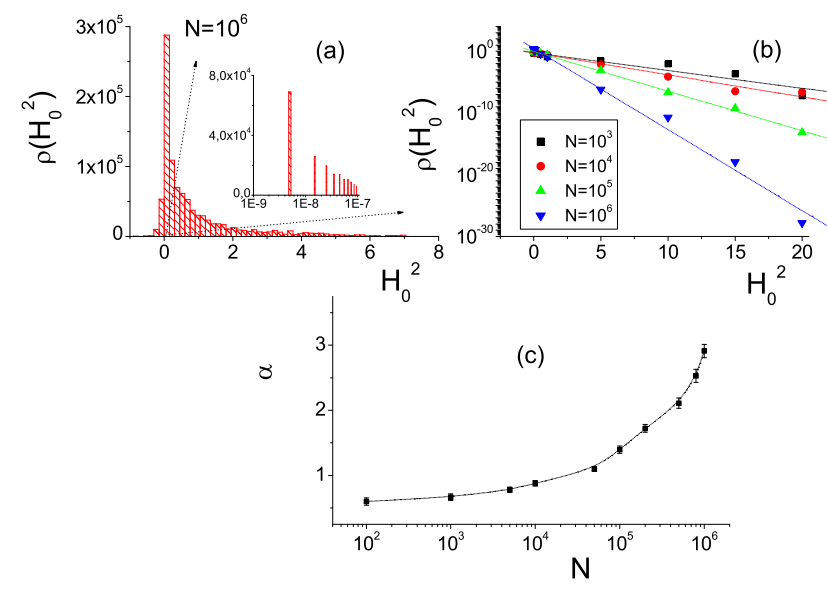

FIG. 2: (Color online) In graph a) we present the $H_{0}^{2}$ histogram for the accepted solutions. In graph b) we show the histogram $\rho\left(H_{0}^{2}\right)$ of $H_{0}^{2}$, for four different numbers of physically accepted solutions, where the corresponding curves have been normalized to area 1. The dotted lines correspond to linear fits. In graph c) we depict the dependence of exponent $\alpha$, of the exponential form (13), on the physically accepted solution number $N$. Numerical extrapolation gives $\alpha \rightarrow \infty$ for $N \rightarrow \infty$. All graphs refer to the stationary case with full bulk potential.

space of the parameter space, which becomes thinner for "larger" $H_{0}$, resulting eventually to a sub-surface.

The natural arising question is what factors affect the exponent $\alpha$ of relation (13). We can show numerically that it is determined by the statistics and increases with the number of solutions $N$. In fig. 2 b we depict $\rho\left(H_{0}^{2}\right)$ vs $H_{0}^{2}$, for four different- $N$ cases. We clearly see that the exponential form decays faster for larger $N$. In fig. 2 c we present the values of the exponent $\alpha$, arising from numerical fits of the form of (13), for various $N$ values. Indeed, $\alpha$ increases with $N$, and the increasing rate is robust although quite slow. Numerical extrapolation gives $\alpha \rightarrow \infty$ for $N \rightarrow \infty$. This is a favoring result since it implies that covering continuously and uniformly the parameter space, the physically accepted solutions compose for $\rho\left(H_{0}^{2}\right)$ the $\delta$-function $\delta\left(H_{0}^{2}\right)$. Solutions with large $H_{0}$ are not impossible, bur arise from a sub-surface of smaller dimensionality. In the continuum limit, large $H_{0}$ solutions are of zero measure while the ones with $H_{0} \approx 0$ are by far the most probable (note that although we are using $M_{5}$ units, the presence of $\delta\left(H_{0}^{2}\right)$ leads to $H_{0} \approx 0$ in every units). As it is well known, $H_{0}^{2}$ in our Universe is related to the (effective in a $5 D$ model) $4 D$ cosmological constant of our world. Since $\left|\lambda_{\text {eff }}\right| \lesssim\left|H_{0}^{2}\right|$ we finally acquire $\rho\left(\left|\lambda_{\text {eff }}\right|\right) \approx \delta\left(\left|\lambda_{\text {eff }}\right|\right)$, compatible with observations.

The distribution for $\lambda_{\text {eff }}$ obtained above is free of both explicit and implicit fine-tuning, since we have not used an explicit relation for $\lambda_{\text {eff }}$ in terms of the model parameters and then force this expression to be small. On the contrary we have just searched for general parameter octads that correspond to solutions and for these octads we have calculated $H_{0}$. In fig. 3 we present the histograms of the model parameters that correspond to $N=10^{6}$ accepted solutions, for the stationary case with full bulk potential. As we can clearly see, apart from a small ten-

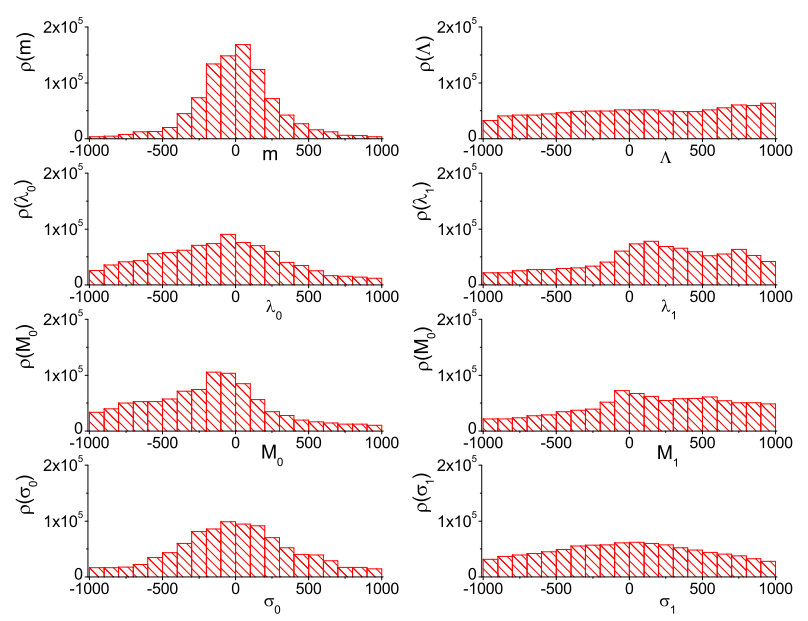

FIG. 3: (Color online) The histograms of the model parameters $m, \Lambda, \lambda_{0}, \lambda_{1}, M_{0}, M_{1}, \sigma_{0}, \sigma_{1}$, that correspond to $N=10^{6}$ accepted solutions, for the stationary case with full bulk potential.

dency of $m$ to small values, there is no specific preference to particular values. To quantify this behavior we calculate the correlation dimension of the sub-space of the parameter space that corresponds to accepted solutions. Denoting by $\vec{r}_{i j}$ the 8D Euclidean distances between two solution-octads $i$ and $j$ :

$$
\begin{array}{r}
\left|\vec{r}_{i j}\right|^{2}=\left(m_{i}-m_{j}\right)^{2}+\left(\Lambda_{i}-\Lambda_{j}\right)^{2}+\left(\lambda_{0 i}-\lambda_{0 j}\right)^{2}+ \\
+\left(\lambda_{1 i}-\lambda_{1 j}\right)^{2}+\left(M_{0 i}-M_{0 j}\right)^{2}+\left(M_{1 i}-M_{1 j}\right)^{2}+ \\
+\left(\sigma_{0 i}-\sigma_{0 j}\right)^{2}+\left(\sigma_{1 i}-\sigma_{1 j}\right)^{2}
\end{array}
$$

we use the standard approach introduced by Grassberger and Procaccia [22] to calculate the correlation integral

$$
C(r)=\frac{2}{N(N-1)} \sum_{i<j}^{N} \Theta\left(r-\left|\vec{r}_{i j}\right|\right) \propto r^{\beta}
$$

in order to obtain the correlation dimension $\beta$. In fig. 4 we depict $C(r)$ versus $r$, in the region of intermediate $r$ where the statistics allows for the power-law form of relation (15). We observe that it clearly follows (15) with exponent $\beta=7.2 \pm 0.3$. Such a value (within statistical errors) is very close to the value $\beta_{0}=8$ expected for a complete covering of the $8 \mathrm{D}$ space. To estimate the effect of the finite statistics we have repeated the calculations 


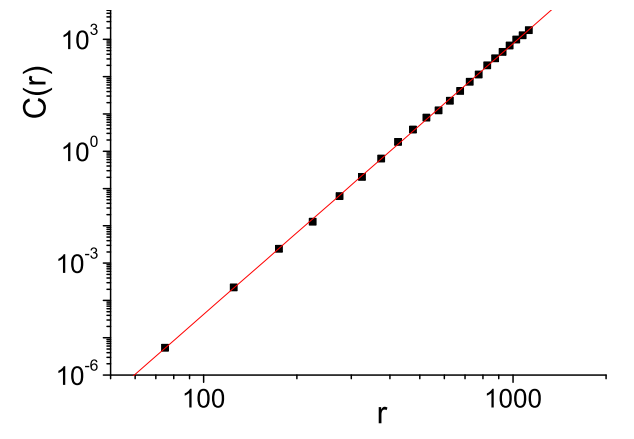

FIG. 4: (Color online) $C(r)$ versus $r$, in the region of intermediate $r$ 's. It follows the power-law form of (15), with exponent $\beta=7.2 \pm 0.3$.

using the same number of random octads within the corresponding hypercube (i.e without taking into account any constraints induced by the requirement of physically accepted solutions), and we have found a typical value for the correlation dimension $\tilde{\beta} \approx 7.4 \pm 0.2$. Therefore, our results should be compared with this value and not with the value $\beta_{0}=8$. Thus, based on this fact, we safely conclude that the obtained $H_{0}^{2}$-histogram and $\lambda_{\text {eff- }}$ distribution, are fine-tuning-free.

In the above analysis we have been restricted to stationary solutions of the form (8) without bothering about time evolution, since in this case $H_{0}=H_{0}(0)=$ const. by construction. The question is what can be said about the full dynamics of equations (3) and (4), where $H_{0}$ can be varying. Fortunately, the solutions of the full dynamics seem to consist of stationary or slowly varying ones [14, 15]. In such an evolution $H_{0}(t)$ can change by at most one order of magnitude. This behavior justifies the above stationary treatment, since the obtained results for $t=0$ will not change qualitatively for $t>0$.

In order to present this behavior in a more transparent way we investigate numerically the full dynamical system (3), (4) and (5), that is without imposing the ansatz (8). In particular, we first perform the procedure described above for $t=0$ (or equivalently for initial brane propertime $\tau=0$ ), in order to extract $N=5 \times 10^{4}$ parameter octads that correspond to solutions, and for these octads we calculate $H_{0}(0)$ and $\rho\left(H_{0}^{2}(0)\right)$. For these parameter octads we evolve the system according to (3) and (5), and thus for each brane proper-time we obtain the corresponding histogram $\rho\left(H_{0}^{2}(\tau)\right)$. We mention that for a significant portion of the initially acquired solutions $(\approx 20 \%)$, the subsequent time evolution leads to divergences, since the singularities transit from outside to inside the interbrane space. This behavior was also found in [15]. Thus these parameter octads are neglected.

In fig. [5w we present the initial histogram $\rho\left(H_{0}^{2}(0)\right)$ (upper graph) and the histogram $\rho\left(H_{0}^{2}(\tau)\right)$ for $\tau=10^{3}$ in $M_{5}$ units (lower graph). As we observe, indeed the vari-

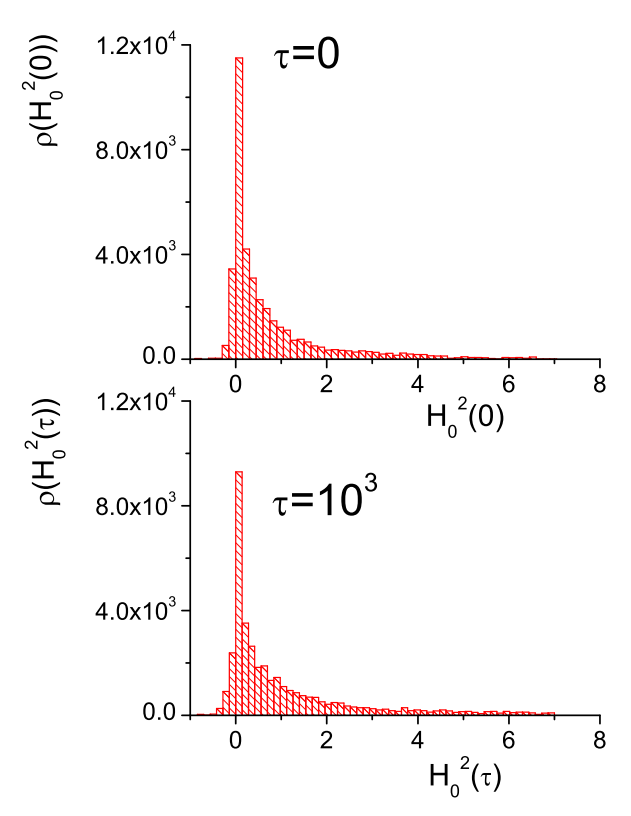

FIG. 5: (Color online) The initial histogram $\rho\left(H_{0}^{2}(0)\right)$ (upper graph) and the histogram $\rho\left(H_{0}^{2}(\tau)\right)$ for $\tau=10^{3}$ in $M_{5}$ units (lower graph), for $N=5 \times 10^{4}$ physically accepted solutions of the full dynamical system (3), (4) and (5), with full brane and bulk potentials.

ation of $H_{0}(\tau)$ from its initial value $H_{0}(0)$ is less than an order of magnitude for the significant portion of cases. Note also that this variation can be either an increase or a decrease. Thus, in summary, the final histogram is only slightly wider. To quantify this deformation, in fig. 6 we present the aforementioned histograms in logarithmic scale, normalized to area 1 . As we can see, time evolu-

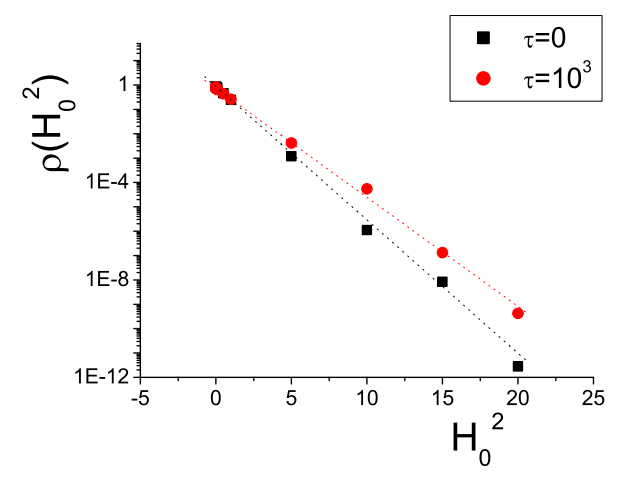

FIG. 6: (Color online) The initial histogram $\rho\left(H_{0}^{2}(0)\right)$ and the histogram $\rho\left(H_{0}^{2}(\tau)\right)$ for $\tau=10^{3}$ in $M_{5}$ units, for $N=5 \times$ $10^{4}$ physically accepted solutions of the full dynamical system (3), (4) and (5), with full brane and bulk potentials. The histograms have been normalized to area 1 and the dotted lines correspond to linear fits.

tion does not change our results significantly. Thus, in the limit $N \rightarrow \infty$ the $\delta$-function-form $\rho\left(\left|\lambda_{\text {eff }}\right|\right) \approx \delta\left(\left|\lambda_{\text {eff }}\right|\right)$ 
will be preserved.

One enlightening interpretation of the aforementioned result is obtained by a parallelism with the equilibration mechanism of an isolated macroscopic system through entropy maximization. "Peculiar" states are not impossible, but there is extremely small probability for their realization. In a similar way, in braneworld models, the large majority (in the continuum limit "almost" all) of the parametric combinations that correspond to physically accepted solutions, lead to an extremely small cosmological constant.

In conclusion, using a statistical approach, we have shown that in the context of a general Braneworld scenario the acceptable solutions of the Einstein equations are compatible with a $\delta$-function distribution for the $4 D$ effective cosmological constant. Additionally, within this treatment, $\lambda_{\text {eff }}$ is small and remains so even if the model parameters vary by many orders of magnitudes due to successive cosmological phase transitions. Of course the above analysis cannot at all act as a solution to the cosmological constant problem. It only provides some argument for its possible elaboration in statistical terms.

\section{Acknowledgment}

We thank G. Felder, A. Frolov, G. Kofinas and N. Tetradis for useful discussions.

[1] S. Weinberg, Rev. Mod. Phys. 61, 1 (1989).

[2] A. Vilenkin, Contribution to Universe or Multiverse, Cambridge University Press (2007).

[3] B. Zumino, Nucl. Phys. B 89, 535 (1975).

[4] Z. Kakushadze, Nucl. Phys. B 589, 75 (2000); Y. Aghababaie, C. P. Burgess, S. L. Parameswaran and F. Quevedo, Nucl. Phys. B 680, 389 (2004); A. J. Tolley, C. P. Burgess, D. Hoover and Y. Aghababaie, JHEP
0603, 091 (2006)

[5] O. DeWolfe, D. Z. Freedman, S. S. Gubser and A. Karch, Phys. Rev. D 62, 046008 (2000).

[6] E. Witten, Sources and detection of dark matter and dark energy in the universe, Marina del Rey 2000; R. Bousso and J. Polchinski, JHEP 0006, 006 (2000).

[7] S. W. Hawking, Phys. Lett. B 134, 403 (1984); S. Coleman, Nucl. Phys. B 310, 643 (1988).

[8] I. P. Neupane, B. M. N. Carter, Phys. Lett. B 638, 94 (2006).

[9] H. Martel, P. R. Shapiro and S. Weinberg, Astrophys. J. 492, 29 (1998).

[10] S. Perlmutter et al., Astrophys. J. 517, 565 (1999).

[11] L. Randall and R. Sundrum, Phys. Rev. Lett. 83, 3370 (1999); L. Randall and R. Sundrum, Phys. Rev. Lett. 83, 4690 (1999).

[12] S. Kachru, M. Schulz and E. Silverstein, Phys. Rev. D f62, 045021 (2000); S. P. de Alwis, Nucl. Phys. B 597, 263 (2001); S. H. Henry Tye and I. Wasserman, Phys. Rev. Lett. 86, 1682 (2001); A. Kehagias and K. Tamvakis, Mod. Phys. Lett. A 17, 1767 (2002); S. Mukohyama and L. Randall, Phys. Rev. Lett. 92, 211302 (2004).

[13] S. Forste et al., Phys. Lett. B 481360 (2000); S. Forste et al., JHEP 0009, 034 (2000).

[14] N. Tetradis, Phys. Lett. B 509, 307 (2001).

[15] J. Martin, G. N. Felder, A. V. Frolov, M. Peloso and L. A. Kofman, Phys. Rev. D 69, 084017 (2004).

[16] W. D. Goldberger and M. B. Wise, Phys. Rev. Lett. 83, 4922 (1999).

[17] E. N. Saridakis, JCAP 0804, 020 (2008).

[18] E. N. Saridakis, Nucl. Phys. B 808, 224 (2009).

[19] P. Kanti, K. A. Olive and M. Pospelov, Phys. Lett. B 481, 386 (2000).

[20] E. Flanagan, S.-H. H. Tye and I. Wasserman, Phys. Lett. B 522, 155 (2001).

[21] P. Schmelcher and F. K. Diakonos, Phys. Rev. Lett. 78, 4733 (1997); F. K. Diakonos, P. Schmelcher and O. Biham, Phys. Rev. Lett. 81, 4349 (1998).

[22] P. Grassberger and I. Procaccia, Physica D 9, 189 (1983). 